Georgian Mathematical Journal

Volume 12 (2005), Number 2, 377-387

\title{
MAPPING PROPERTIES OF INTEGRAL OPERATORS OF LEVY TYPE
}

\author{
THOMAS RUNST AND ABDELLAH YOUSSFI
}

\begin{abstract}
We study the boundedness and compactness of a special class of integral operators defined on generalized Sobolev spaces.

2000 Mathematics Subject Classification: 47G10, 47G20, 46E35, $47 \mathrm{D} 07$.

Key words and phrases: Integral operator, Bessel potential spaces, Markov process, mapping properties.
\end{abstract}

\section{Introduction and the Main Results}

Let us consider the integral operator $S$,

$$
S u(x)=\int_{\mathbb{R}^{n} \backslash\{0\}}\left[u(x+z)-u(x)-\sum_{j=1}^{n} z_{j} \frac{\partial u}{\partial x_{j}}(x)\right] K(x, z) d \mu(z), \quad x \in \mathbb{R}^{n},
$$

where the kernel $K$ is defined on $\mathbb{R}^{n} \times \mathbb{R}^{n}$ and satisfies the following growth conditions:

- (H1): $K \in L^{\infty}\left(\mathbb{R}^{n} \times \mathbb{R}^{n}\right)$,

- (H2): for fixed $0<\theta_{0}<1$, we suppose that $H\left(\theta_{0}\right)<+\infty$, where

$$
H\left(\theta_{0}\right)=\sup _{x, z \in \mathbb{R}^{n}} \int_{\mathbb{R}^{n}}|K(x+h, z)-K(x, z)|^{2} \frac{d h}{|h|^{n+2 \theta_{0}}} .
$$

Furthermore, the Radon measure $d \mu(z)$ on $\mathbb{R}^{n} \backslash\{0\}$ satisfies the moment condition:

- (H3): $\int_{0<|z| \leq 1}|z|^{\alpha} d \mu(z)+\int_{|z| \geq 1}|z| d \mu(z)<+\infty$, where $1 \leq \alpha \leq 2$ holds.

Our first mapping result deals with the generalized Sobolev spaces $H_{p}^{s}\left(\mathbb{R}^{n}\right)$ of functions defined on $\mathbb{R}^{n}$.

Theorem 1. Suppose that (H1), (H2) and (H3) hold. Then

$$
S: H_{p}^{\theta+\alpha}\left(\mathbb{R}^{n}\right) \rightarrow H_{p}^{\theta}\left(\mathbb{R}^{n}\right)
$$

is a bounded operator for all $1<p<\infty$ and all $0 \leq \theta \leq \theta_{0}$.

In our second result we consider the case of bounded connected domains $\Omega$ in $\mathbb{R}^{n}$ with smooth boundary $\partial \Omega$. 
Theorem 2. Let $K \in L^{\infty}\left(\mathbb{R}^{n} \times \mathbb{R}^{n}\right)$ with $K(x, z)=0$ if $x+z \notin \bar{\Omega}$. Suppose that $(\mathrm{H} 2)$ and $(\mathrm{H} 3)$ are satisfied with $0<\theta_{0}<1$ and $1 \leq \alpha \leq 2$, respectively. Then

$$
S: H_{p}^{\alpha+\theta}(\Omega) \rightarrow H_{p}^{\theta}(\Omega)
$$

is bounded for $0 \leq \theta \leq \theta_{0}$ and all $1<p<+\infty$. Moreover, if $\frac{n}{p}+1<\alpha$, then

$$
S: H_{p}^{\alpha+\theta}(\Omega) \rightarrow H_{p}^{\theta}(\Omega)
$$

is compact for all $0 \leq \theta<\theta_{0}$.

Note that the condition $K(x, z)=0$ for $x+z \notin \bar{\Omega}$ implies that our integral operator can be interpreted as a mapping acting on functions $u$ which are defined in $\bar{\Omega}$.

Operators of such a type play an important role in the theory of Waldenfels operators $W=P+S$, where $P$ is a second-order elliptic partial differential operator of the type

$$
P u(x)=\sum_{i, j=1}^{n} a_{i, j}(x) \frac{\partial^{2} u}{\partial x_{i} \partial x_{j}}(x)+\sum_{i=1}^{n} b_{i}(x) \frac{\partial u}{\partial x_{i}}(x)+c(x) u(x), \quad x \in \Omega,
$$

see [1] and [4]. In particular, the Levy operator $S$ occurs as a compact perturbation.

The paper is organized as follows. First we recall some properties of spaces of type $H_{p}^{s}$. In Section 3, we prove Theorem 1 and then Theorem 2 in Section 4.

\section{Function Spaces}

In general, all functions, distributions, etc. are defined on the Euclidean space $\mathbb{R}^{n}$. As usual $\mathcal{S}=\mathcal{S}\left(\mathbb{R}^{n}\right)$ denotes the Schwartz space of test functions, $\mathcal{S}^{\prime}=\mathcal{S}^{\prime}\left(\mathbb{R}^{n}\right)$ is its dual. For $f \in \mathcal{S}^{\prime}, \hat{f}$ denotes the Fourier transform of $f$.

To define the Bessel potential space $H_{p}^{s}=H_{p}^{s}\left(\mathbb{R}^{n}\right)$, we make use of the Fourieranalytic approach.

Throughout the paper let $\psi$ in $\mathcal{S}\left(\mathbb{R}^{n}\right)$ be fixed so that $\hat{\psi}$ be supported by the set $\left\{\xi \in \mathbb{R}^{n}: \frac{1}{2} \leq|\xi| \leq 2\right\}$ and

$$
\sum_{j \in \mathbb{Z}} \hat{\psi}\left(2^{j} \xi\right)=1 \quad \text { for } \quad \xi \neq 0
$$

Define $\varphi$ by

$$
\hat{\varphi}(\xi)=1-\sum_{j \geq 1} \hat{\psi}\left(2^{-j} \xi\right)
$$

and denote now by $\Delta_{j}(j \in \mathbb{N})$ the convolution operator with symbol $\hat{\psi}\left(2^{-j} \xi\right)$.

For $s \in \mathbb{R}$ and $1<p<+\infty$, the generalized Sobolev space or the Bessel potential space $H_{p}^{s}=H_{p}^{s}\left(\mathbb{R}^{n}\right)$ is a subspace of $\mathcal{S}^{\prime}\left(\mathbb{R}^{n}\right)$ given by the norm

$$
\|g\|_{H_{p}^{s}}=\|\varphi * g\|_{p}+\left\|\left[\sum_{j \geq 1} 4^{s j}\left|\Delta_{j} g\right|^{2}\right]^{\frac{1}{2}}\right\|_{p}<+\infty
$$


and for $1 \leq q \leq \infty$, the Besov space $B_{p, q}^{s}=B_{p, q}^{s}\left(\mathbb{R}^{n}\right)$ is a subspace of $\mathcal{S}^{\prime}\left(\mathbb{R}^{n}\right)$ with the norm

$$
\|g\|_{B_{p, q}^{s}}=\|\varphi * g\|_{p}+\left[\sum_{j \geq 1} 2^{s j q}\left\|\Delta_{j} g\right\|_{p}^{q}\right]^{\frac{1}{q}}<+\infty .
$$

Note that if $s<s^{\prime}$ holds, then $H_{p}^{s} \subset B_{p, \infty}^{s}$ and $B_{p, \infty}^{s^{\prime}} \subset H_{p}^{s}$.

Now let $\Omega$ be a bounded connected domain in $\mathbb{R}^{n}$ with smooth boundary $\partial \Omega$, and its closure be given by $\bar{\Omega}=\Omega \cup \partial \Omega$. To define function spaces on $\Omega$ we use restriction arguments. Let $\mathcal{D}^{\prime}(\Omega)$ be the space of distributions on $\Omega$. Then we put

$$
H_{p}^{s}(\Omega)=\left\{f \in \mathcal{D}^{\prime}(\Omega): \quad \exists g \in H_{p}^{s}\left(\mathbb{R}^{n}\right) \text { such that }\left.g\right|_{\Omega}=f\right\}
$$

and

$$
\|f\|_{H_{p}^{s}(\Omega)}=\inf _{\left.g\right|_{\Omega}=f}\|g\|_{H_{p}^{s}} .
$$

In the same way we define the Besov space

$$
B_{p, q}^{s}(\Omega)=\left\{f \in \mathcal{D}^{\prime}(\Omega): \quad \exists g \in B_{p, q}^{s}\left(\mathbb{R}^{n}\right) \text { such that }\left.g\right|_{\Omega}=f\right\}
$$

and

$$
\|f\|_{B_{p ; q}^{s}(\Omega)}=\inf _{\left.g\right|_{\Omega}=f}\|g\|_{B_{p, q}^{s}}
$$

see for example [5] and [3].

For the proof of Theorem 1 we make use of the following characterization of generalized Sobolev spaces $H_{p}^{s}$ (Strichartz' norm) which can be found, for example, in [5, p. 194].

Lemma 1. Let $0<s<1$ and $1<p<+\infty$. Then

$$
N_{p}^{s}(g)=\|g\|_{p}+\left\|L_{s}(g)\right\|_{p}
$$

defines an equivalent norm on $H_{p}^{s}$, where

$$
L_{s}(g)(x)= \begin{cases}\left(\int_{\mathbb{R}^{n}}|g(x+h)-g(x)|^{2} \frac{d h}{|h|^{n+2 s}}\right)^{1 / 2} & \text { if } p \geq 2, \\ \left(\int_{0}^{\infty}\left[\int_{|h| \leq 1}|g(x+t h)-g(x)| d h\right]^{2} \frac{d t}{t^{1+2 s}}\right)^{1 / 2} & \text { if } 1<p \leq 2 .\end{cases}
$$

The second Lemma which will be used in our proof is the following.

Lemma 2. Let $0 \leq s \leq 1,0 \leq \gamma \leq 1,1<p<+\infty$ and set $T_{h}(f)(x)=$ $f(x+h)-f(x)$. Then there exists $C>0$ such that

$$
\left\|T_{h}(f)\right\|_{H_{p}^{\gamma}} \leq C|h|^{s}\|f\|_{H_{p}^{s+\gamma}}
$$

for all $f \in H_{p}^{s+\gamma}$. 
Proof. We are not able to give a reference for this result. For the reader's convenience we prove the assertion.

1) The case $\gamma=0$ :

for $s=0$ the lemma is trivial and for $s=1$ it is known, see [6, pp. 45-46], for example;

for $0<s<1$ we recall that

$$
\left[\int_{\mathbb{R}^{n}}|f(x+h)-f(x)|^{p} d x\right]^{\frac{1}{p}} \leq C|h|^{s}\|f\|_{B_{p, \infty}^{s}} .
$$

Since $H_{p}^{s} \subset B_{p, \infty}^{s}$ the lemma follows.

2) The case $\gamma=1$ :

Note that

$$
\left\|T_{h}(f)\right\|_{H_{p}^{1}} \leq C\left[\sum_{j=1}^{n}\left\|\frac{\partial T_{h}(f)}{\partial x_{j}}\right\|_{p}+\left\|T_{h}(f)\right\|_{p}\right]
$$

and it follows from 1) that

$$
\left\|T_{h}(f)\right\|_{p}+\left\|\frac{\partial T_{h}(f)}{\partial x_{j}}\right\|_{p} \leq C|h|^{s}\left[\left\|\frac{\partial f}{\partial x_{j}}\right\|_{H_{p}^{s}}+\|f\|_{p}\right]
$$

and hence

$$
\left\|T_{h}(f)\right\|_{H_{p}^{1}} \leq C|h|^{s}\|f\|_{H_{p}^{s+1}} .
$$

3) From 1) and 2) we obtain that $T_{h}$ is a bounded linear operator from $H_{p}^{s}$ into $L^{p}$ and from $H_{p}^{s+1}$ into $H_{p}^{1}$, respectively.

Then by interpolation arguments we obtain that $T_{h}$ is bounded from $H_{p}^{s+\gamma}$ into $H_{p}^{\gamma}$ for all $0 \leq \gamma \leq 1$ and the norm of $T_{h}$ is bounded by $C|h|^{s}$.

Lemma 3. Let $0<\theta_{0}<1$. There exists $C_{1}=C_{1}\left(\theta_{0}\right)>0$ such that

$$
\sup _{x, h \in \mathbb{R}^{n}}\left[\frac{|g(x+h)-g(x)|}{|h|^{\theta_{0}}}\right] \leq C_{1}\left[\sup _{x \in \mathbb{R}^{n}}\left[\int_{\mathbb{R}^{n}}|g(x+h)-g(x)|^{2} \frac{d h}{|h|^{n+2 \theta_{0}}}\right]^{\frac{1}{2}}+\|g\|_{\infty}\right]
$$

for all $g \in L^{\infty}\left(\mathbb{R}^{n}\right)$. Moreover, for every $0 \leq \theta<\theta_{0}$, there exists a finite constant $C_{2}=C_{2}\left(\theta_{0}\right)>0$ such that

$$
\left.\int_{\mathbb{R}^{n}}|g(x+h)-g(x)|^{2} \frac{d h}{|h|^{n+2 \theta}}\right]^{\frac{1}{2}} \leq C_{1}\left[\sup _{x, h \in \mathbb{R}^{n}} \frac{|g(x+h)-g(x)|}{|h|^{\theta_{0}}}+\|g\|_{\infty}\right]
$$

for all $g \in L^{\infty}\left(\mathbb{R}^{n}\right)$.

In particular, if $K \in L^{\infty}\left(\mathbb{R}^{n} \times \mathbb{R}^{n}\right)$, then $H\left(\theta_{0}\right)<+\infty$ implies $H(\theta)<+\infty$ for all $0 \leq \theta<\theta_{0}$ and

$$
|g(x+h)-g(x)| \leq C|h|^{\theta_{0}}
$$

for all $x, h \in \mathbb{R}^{n}$. 
Proof. Indeed, we have

$$
\|g\|_{B_{\infty}^{\theta_{0}}, \infty}=\|g\|_{\infty}+\sup _{x, h \in \mathbb{R}^{n}} \frac{|g(x+h)-g(x)|}{|h|^{\theta_{0}}}
$$

and

$$
\|g\|_{B_{\infty, 2}^{\theta_{0}}}=\|g\|_{\infty}+\sup _{x \in \mathbb{R}^{n}}\left[\int_{\mathbb{R}^{n}}|g(x+h)-g(x)|^{2} \frac{d h}{\mid h^{n+2 \theta_{0}}}\right]^{\frac{1}{2}},
$$

see [5] or [3] for example. The embeddings $B_{\infty, 2}^{\theta_{0}} \subset B_{\infty, 2}^{\theta} \subset B_{\infty, \infty}^{\theta}$ finish the proof.

\section{Proof of Theorem 1}

To prove Theorem 1 we show first the boundedness of $S$ from $H_{p}^{\alpha}\left(\mathbb{R}^{n}\right)$ into $L^{p}\left(\mathbb{R}^{n}\right)$ (the case $\theta=0$ ) and after that we prove that there exists a positive constant $C$ such that

$$
\left\|L_{\theta}(S u)\right\|_{p} \leq C\|u\|_{H_{p}^{\alpha+\theta}}
$$

holds for all $u \in H_{p}^{\alpha+\theta}\left(\mathbb{R}^{n}\right)$ and $0<\theta \leq \theta_{0}$. This implies

$$
\|S u\|_{H_{p}^{\theta}} \leq C\|u\|_{H_{p}^{\theta+\alpha}} .
$$

Indeed, $H\left(\theta_{0}\right)<+\infty$ implies $H(\theta)<+\infty$ by Lemma 3 .

3.1. $L^{p}$-boundedness. To prove the $L^{p}$-boundedness, we write $S u(x)=S_{1} u(x)$ $+S_{2} u(x)$, where

$$
\begin{aligned}
& S_{1} u(x)=\int_{|z| \geq 1}\left[u(x+z)-u(x)-\sum_{j=1}^{n} z_{j} \frac{\partial u}{\partial x_{j}}(x)\right] K(x, z) d \mu(z), \\
& S_{2} u(x)=\int_{|z| \leq 1}\left[u(x+z)-u(x)-\sum_{j=1}^{n} z_{j} \frac{\partial u}{\partial x_{j}}(x)\right] K(x, z) d \mu(z) .
\end{aligned}
$$

First observe that

$$
\left|S_{1} u(x)\right| \leq\|K\|_{\infty}\left[g_{1}(x)+g_{2}(x)\right]
$$

where

$$
g_{1}(x)=\int_{|z| \geq 1}|u(x+z)-u(x)| d \mu(z)
$$

and

$$
g_{2}(x)=\sum_{j=1}^{n} \frac{\partial u}{\partial x_{j}}(x) \int_{|z| \geq 1}\left|z_{j}\right| d \mu(z)
$$

Hence

$$
\left\|g_{1}\right\|_{p} \leq \int_{|z| \geq 1}\left[\int_{\mathbb{R}^{n}}|u(x+z)-u(x)|^{p} d x\right]^{\frac{1}{p}} d \mu(z)
$$


and

$$
\left\|g_{2}\right\|_{p} \leq \sum_{j=1}^{n}\left\|\frac{\partial u}{\partial x_{j}}\right\|_{p}\left[\sum_{j=1}^{n} \int_{|z| \geq 1}\left|z_{j}\right| d \mu(z)\right] .
$$

Combining now (H3), Lemma 2 with $s=1$ and $\gamma=0$, we obtain

$$
\left\|S_{1} u\right\|_{p} \leq C\|K\|_{\infty}\|u\|_{H_{p}^{1}} \int_{|z| \geq 1}|z| d \mu(z) .
$$

Next we give an estimate for $\left\|S_{2} u\right\|_{p}$. We write

$$
u(x+z)-u(x)-\sum_{j=1}^{n} z_{j} \frac{\partial u}{\partial x_{j}}(x)=\sum_{j=1}^{n} z_{j} \int_{0}^{1}\left[\frac{\partial u}{\partial x_{j}}(x+t z)-\frac{\partial u}{\partial x_{j}}(x)\right] d t,
$$

and it follows that

$$
\left|S_{2} u(x)\right| \leq\|K\|_{\infty} \sum_{j=1}^{n} \int_{0}^{1} \int_{|z| \leq 1}|z|\left|\frac{\partial u}{\partial x_{j}}(x+t z)-\frac{\partial u}{\partial x_{j}}(x)\right| d \mu(z) d t .
$$

Hence

$$
\left\|S_{2} u\right\|_{p} \leq C \sum_{j=1}^{n}\|K\|_{\infty} \int_{0}^{1} \int_{|z| \leq 1}|z|\left[\int_{\mathbb{R}^{n}}\left|\frac{\partial u}{\partial x_{j}}(x+t z)-\frac{\partial u}{\partial x_{j}}(x)\right|^{p} d x\right]^{\frac{1}{p}} d \mu(z) d t .
$$

Applying now Lemma 2 with $s=\alpha-1$ and $\gamma=0$, we obtain

$$
\left[\int_{\mathbb{R}^{n}}\left|\frac{\partial u}{\partial x_{j}}(x+t z)-\frac{\partial u}{\partial x_{j}}(x)\right|^{p} d x\right]^{\frac{1}{p}} \leq C|t z|^{\alpha-1}\left\|\frac{\partial u}{\partial x_{j}}\right\|_{H_{p}^{\alpha-1}} \leq C|t z|^{\alpha-1}\|u\|_{H_{p}^{\alpha}}
$$

Thus

$$
\left\|S_{2} u\right\|_{p} \leq C\|K\|_{\infty}\|u\|_{H_{p}^{\alpha}} \int_{|z| \leq 1}|z|^{\alpha} d \mu(z)
$$

3.2. Estimation of $\left\|L_{\theta}(S u)\right\|_{p}$. In the following, we consider the case where $p \geq 2$ holds. The other case can be handled similarly. We are to prove that $L_{\theta}(S u) \in L^{p}$, where

$$
L_{\theta}(S u)(x)=\left[\int \frac{|S u(x+h)-S u(x)|^{2}}{|h|^{n+2 \theta}} d h\right]^{\frac{1}{2}} .
$$

As above we put $S u=S_{1} u+S_{2} u$, where

$$
\begin{aligned}
& S_{1} u(x)=\int_{|z| \geq 1}\left[u(x+z)-u(x)-\sum_{j=1}^{n} z_{j} \frac{\partial u}{\partial x_{j}}(x)\right] K(x, z) d \mu(z), \\
& S_{2} u(x)=\int_{|z| \leq 1}\left[u(x+z)-u(x)-\sum_{j=1}^{n} z_{j} \frac{\partial u}{\partial x_{j}}(x)\right] K(x, z) d \mu(z) .
\end{aligned}
$$


A) Estimation of $L_{\theta}\left(S_{1} u\right)$. First we write $S_{1} u(x+h)-S_{1} u(x)$ in the following form:

$$
\begin{aligned}
& S_{1} u(x+h)-S_{1} u(x) \\
& =\int_{|z| \geq 1}[u(x+h+z)-u(x+z)+u(x)-u(x+h)] K(x+h, z) d \mu(z) \\
& \quad+\int_{|z| \geq 1}[u(x+z)-u(x)][K(x+h, z)-K(x, z)] d \mu(z) \\
& \quad+\sum_{j=1}^{n}\left[\frac{\partial u}{\partial x_{j}}(x)-\frac{\partial u}{\partial x_{j}}(x+h)\right] \int_{|z| \geq 1} z_{j} K(x+h, z) d \mu(z) \\
& \quad+\sum_{j=1}^{n} \frac{\partial u}{\partial x_{j}}(x) \int_{|z| \geq 1} z_{j}[K(x, z)-K(x+h, z)] d \mu(z) \\
& =A_{1}(x, h)+A_{2}(x, h)+A_{3}(x, h)+A_{4}(x, h) .
\end{aligned}
$$

1) $A_{1}(x, h)$ :

We have

$$
A_{1}(x, h)=\int_{|z| \geq 1}\left(\int_{0}^{1} \sum_{j=1}^{n}\left[\frac{\partial u}{\partial x_{j}}(x+h+t z)-\frac{\partial u}{\partial x_{j}}(x+t z)\right] z_{j} d t\right) K(x+h, z) d \mu(z)
$$

which implies

$$
\left[\int_{\mathbb{R}^{n}}\left[\int_{\mathbb{R}^{n}}\left|A_{1}(x, h)\right|^{2} \frac{d h}{|h|^{n+2 \theta}}\right]^{\frac{p}{2}} d x\right]^{\frac{1}{p}} \leq C \sum_{j=1}^{n}\left\|L_{\theta}\left(\frac{\partial u}{\partial x_{j}}\right)\right\|_{p}\|K\|_{\infty}\left[\int_{|z| \geq 1}|z| d \mu(z)\right] .
$$

2) $A_{2}(x, h)$ :

In this case we get

$$
\begin{aligned}
& {\left[\int_{\mathbb{R}^{n}}\left|A_{2}(x, h)\right|^{2} \frac{d h}{|h|^{n+2 \theta}}\right]^{\frac{1}{2}}} \\
& \leq \int_{|z| \geq 1}|u(x+z)-u(x)| \int_{\mathbb{R}^{n}}\left[|K(x+h, z)-K(x, z)|^{2} \frac{d h}{\mid h^{n+2 \theta}}\right]^{\frac{1}{2}} d \mu(z) \\
& \leq H(\theta) \int_{|z| \geq 1}|u(x+z)-u(x)| d \mu(z)
\end{aligned}
$$

and

$$
\left[\int_{\mathbb{R}^{n}}\left[\int_{\mathbb{R}^{n}}\left|A_{2}(x, h)\right|^{2} \frac{d h}{|h|^{n+2 \theta}}\right]^{\frac{p}{2}} d x\right]^{\frac{1}{p}} \leq C H(\theta)\|u\|_{p}\left[\int_{|z| \geq 1}|z| d \mu(z)\right] .
$$


3) $A_{3}(x, h)$ :

It is clear that

$$
\left[\int_{\mathbb{R}^{n}}\left|A_{3}(x, h)\right|^{2} \frac{d h}{|h|^{n+2 \theta}}\right]^{\frac{1}{2}} \leq \sum_{j=1}^{n}\left[\|K\|_{\infty} L_{\theta}\left(\frac{\partial u}{\partial x_{j}}\right)(x)\right]\left[\int_{|z| \geq 1}|z| d \mu(z)\right]
$$

and hence

$$
\left[\int_{\mathbb{R}^{n}}\left[\int_{\mathbb{R}^{n}}\left|A_{3}(x, h)\right|^{2} \frac{d h}{|h|^{n+2 \theta}}\right]^{\frac{p}{2}} d x\right]^{\frac{1}{p}} \leq \sum_{j=1}^{n}\left[\|K\|_{\infty}\left\|L_{\theta}\left(\frac{\partial u}{\partial x_{j}}\right)\right\|_{p}\right]\left[\int_{|z| \geq 1}|z| d \mu(z)\right] .
$$

4) $A_{4}(x, h)$ :

In the last case we obtain

$$
\left[\int_{\mathbb{R}^{n}}\left[\int_{\mathbb{R}^{n}}\left|A_{4}(x, h)\right|^{2} \frac{d h}{|h|^{n+2 \theta}}\right]^{\frac{p}{2}} d x\right]^{\frac{1}{p}} \leq H(\theta)\left[\int_{|z| \geq 1}|z| d \mu(z)\right] \sum_{j=1}^{n}\left\|\frac{\partial u}{\partial x_{j}}\right\|_{p} .
$$

Summing up we get

$$
\begin{aligned}
\left\|L_{\theta}\left(S_{1} u\right)\right\|_{p} \leq & {\left[\|K\|_{\infty}+H(\theta)\right]\left[\int_{|z| \geq 1}|z| d \mu(z)\right] } \\
& \times\left[\|u\|_{p}+\sum_{j=1}^{n}\left\|L_{\theta}\left(\frac{\partial u}{\partial x_{j}}\right)\right\|_{p}+\sum_{j=1}^{n}\left\|\frac{\partial u}{\partial x_{j}}\right\|_{p}\right] .
\end{aligned}
$$

We have

$$
\left\|\frac{\partial u}{\partial x_{j}}\right\|_{p} \leq C\|u\|_{H_{p}^{1}}
$$

and

$$
\left\|L_{\theta}\left(\frac{\partial u}{\partial x_{j}}\right)\right\|_{p} \leq C\|u\|_{H_{p}^{\theta+1}}
$$

It follows finally that

$$
\left\|L_{\theta}\left(S_{1} u\right)\right\|_{p} \leq C\left[\|K\|_{\infty}+H(\theta)\right]\|u\|_{H_{p}^{\theta+1}} \int_{|z| \geq 1}|z| d \mu(z)
$$

B) Estimation of $L_{\theta}\left(S_{2} u\right)$.

Here we write

$$
S_{2} u(x+h)-S_{2} u(x)=B_{1}(x, h)+B_{2}(x, h),
$$

where

$$
\begin{aligned}
B_{1}(x, h)= & \int_{|z| \leq 1}[u(x+z+h)-u(x+z)-u(x+h)+u(x) \\
& \left.-\sum_{j=1}^{n} z_{j}\left(\frac{\partial u}{\partial x_{j}}(x+h)-\frac{\partial u}{\partial x_{j}}(x)\right)\right] K(x+h, z) d \mu(z)
\end{aligned}
$$


and

$$
B_{2}(x, h)=\int_{|z| \leq 1}\left[u(x+z)-u(x)-\sum_{j=1}^{n} z_{j}\left(\frac{\partial u}{\partial x_{j}}(x)\right)(K(x+h, z)-K(x, z))\right] d \mu(z) .
$$

First we put $f_{t z}^{j}(x)=\frac{\partial u}{\partial x_{j}}(x+t z)-\frac{\partial u}{\partial x_{j}}(x)$ and observe that

$$
\left|B_{1}(x, h)\right| \leq \sum_{j=1}^{n}\|K\|_{\infty} \int_{|z| \leq 1} \int_{0}^{1}|z|\left|f_{t z}^{j}(x+h)-f_{t z}^{j}(x)\right| d t d \mu(z) .
$$

Hence we get

$$
\left[\int_{\mathbb{R}^{n}} \frac{\left|B_{1}(x, h)\right|^{2}}{|h|^{n+2 \theta}} d h\right]^{\frac{1}{2}} \leq \sum_{j=1}^{n}\|K\|_{\infty} \int_{|z| \leq 1}|z| \int_{0}^{1} L_{\theta}\left(f_{t z}^{j}\right)(x) d t d \mu(z) .
$$

Applying now Lemma 2 with $s=\alpha-1$ and $\gamma=\theta$, we obtain

$$
\left\|L_{\theta}\left(f_{t z}^{j}\right)\right\|_{p} \leq C|t z|^{\alpha-1}\left\|\frac{\partial u}{\partial x_{j}}\right\|_{H_{p}^{\theta+\alpha-1}}
$$

and, consequently,

$$
\left[\int_{\mathbb{R}^{n}}\left[\int_{\mathbb{R}^{n}} \frac{\left|B_{1}(x, h)\right|^{2}}{|h|^{n+2 \theta}} d h\right]^{\frac{p}{2}} d x\right]^{\frac{1}{p}} \leq C\|K\|_{\infty} \int_{|z| \leq 1}|z|^{\alpha} d \mu(z) \sum_{j=1}^{n}\left\|\frac{\partial u}{\partial x_{j}}\right\|_{H_{p}^{\theta+\alpha-1}} .
$$

On the other hand, we have

$$
\left|B_{2}(x, h)\right| \leq \sum_{j=1}^{n} \int_{0}^{1} \int_{|z| \leq 1}|z|\left|\frac{\partial u}{\partial x_{j}}(x+t z)-\frac{\partial u}{\partial x_{j}}(x)\right||K(x+h, z)-K(x, z)| d \mu(z) d t .
$$

Using (H2), it follows that

$$
\left[\int_{\mathbb{R}^{n}} \frac{\left|B_{2}(x, h)\right|^{2}}{|h|^{n+2 \theta}} d h\right]^{\frac{1}{2}} \leq \sum_{j=1}^{n} H(\theta) \int_{|z| \leq 1}|z| \int_{0}^{1}\left|\frac{\partial u}{\partial x_{j}}(x+t z)-\frac{\partial u}{\partial x_{j}}(x)\right| d \mu(z) d t .
$$

Using again Lemma 2 with $s=\alpha-1$ and $\gamma=0$, we obtain

$$
\left[\int_{\mathbb{R}^{n}}\left[\int_{\mathbb{R}^{n}} \frac{\left|B_{2}(x, h)\right|^{2}}{|h|^{n+2 \theta}} d h\right]^{\frac{p}{2}} d x\right]^{\frac{1}{p}} \leq C H(\theta) \sum_{j=1}^{n}\left\|\frac{\partial u}{\partial x_{j}}\right\|_{H_{p}^{\alpha-1}}
$$

Therefore we have shown that

$$
\left\|L_{\theta}\left(S_{2} u\right)\right\|_{p} \leq C\left(H(\theta)+\|K\|_{\infty}\right)\|u\|_{H_{p}^{\theta+\alpha}}
$$

holds. 


\section{Proof of Theorem 2}

Step 1: The proof of part 1) follows from Theorem 1 by restriction arguments. Step 2: Let $\frac{n}{p}+1<\alpha \leq 2$. We show that

$$
S: H_{p}^{\alpha}(\Omega) \rightarrow L_{p}(\Omega)
$$

is a compact mapping. We recall that $K(x, z)=0$ if $x+z \notin \bar{\Omega}$. Hence there exists a compact set $M \subset \mathbb{R}^{n}$ such that

$$
S u(x)=\int_{M}\left[u(x+z)-u(x)-\sum_{j=1}^{n} z_{j} \frac{\partial u}{\partial x_{j}}(x)\right] K(x, z) d \mu(z)
$$

can be interpreted as a mapping acting on functions $u$ defined on $\bar{\Omega}$.

Now we introduce for $0<\varepsilon<1$ a family of truncation operators given by

$$
S_{\Phi_{\varepsilon}} u(x)=\int_{M}\left[u(x+z)-u(x)-\sum_{j=1}^{n} z_{j} \frac{\partial u}{\partial x_{j}}(x)\right] K(x, z) \Phi_{\varepsilon}(x, z) d \mu(z),
$$

where $\Phi_{\varepsilon} \in C^{\infty}\left(\bar{\Omega} \times \mathbb{R}^{n}\right)$ such that

$$
\Phi_{\varepsilon}(x, z)=0 \quad \text { if }|x-z| \leq \varepsilon,
$$

and

$$
\Phi_{\varepsilon}(x, z)=1 \quad \text { if } \quad|x-z| \geq 2 \varepsilon .
$$

Furthermore, by Lemma 3 there exists $C_{0}>0$ such that

$$
|K(x, z)-K(y, z)|<C_{0}|x-y|^{\theta_{0}}
$$

holds for all $x, y \in M$ and all $z \in \mathbb{R}^{n}$. Hence we can show that

$$
S_{\Phi_{\varepsilon}}: C^{1}(\bar{\Omega}) \rightarrow C(\bar{\Omega})
$$

is bounded. Since $\frac{n}{p}+1<\alpha \leq 2$, the embedding $H_{p}^{\alpha}(\Omega) \hookrightarrow C^{1}(\bar{\Omega})$ is compact and the embedding $C(\bar{\Omega}) \hookrightarrow L^{p}(\Omega)$ is continuous. We establish that

$$
S_{\Phi_{\varepsilon}}: H_{p}^{\alpha}(\Omega) \rightarrow L_{p}(\Omega)
$$

is compact. As in the proof of Theorem 1, we get

$$
\left\|S_{\Phi_{\varepsilon}} u\right\|_{p} \leq C\|K\|_{\infty}\|u\|_{H_{p}^{\alpha}(\Omega)} .
$$

Furthermore, we have by Lebesgue's Theorem,

$$
S_{\Phi_{\varepsilon}} \rightarrow S \quad \text { as } \varepsilon \downarrow 0
$$

with respect to the operator norm in $\mathcal{L}\left(H_{p}^{\alpha}(\Omega), L_{p}(\Omega)\right)$. Because of the fact that the compact operators are a closed subspace in $\mathcal{L}\left(H_{p}^{\alpha}(\Omega), L_{p}(\Omega)\right)$ it follows that

$$
S: H_{p}^{\alpha}(\Omega) \rightarrow L_{p}(\Omega)
$$

is compact for $\frac{n}{p}+1<\alpha$.

Step 3: Now we can finish the proof of Part 2). From Step 1 we can derive that

$$
S: H_{p}^{\alpha+\theta}(\Omega) \rightarrow H_{p}^{\theta}(\Omega)
$$


is bounded for all $1<p<\infty$ and all $0<\theta<\theta_{0}$. Using the result of Step 2 we have

$$
S: H_{p}^{\alpha}(\Omega) \rightarrow L_{p}(\Omega)
$$

is compact if $\frac{n}{p}+1<\alpha$.

Now we can apply a result concerning the complex interpolation of compact linear operators, see [2], in order to obtain our result. Indeed, $H_{p}^{\alpha}(\Omega)$ is reflexive and it is known that $H_{p}^{\alpha}(\Omega)=\left[L^{p}(\Omega), H_{p}^{\alpha+\theta}(\Omega)\right]_{\sigma}$ where $\sigma=1-\frac{\alpha}{\alpha+\theta}$.

\section{REFERENCES}

1. J. -M. Bony, P. Courrège and P. Priouret, Semi-groupes de Feller sur une variété bord compacte et problèmes aux limites intégro-différentiels du second ordre donnant lieu au principe du maximum. Ann. Inst. Fourier (Grenoble) 18(1968), fasc. 2, 369-521 (1969).

2. M. CWIKEL and N. J. KALTON, Interpolation of compact operators by the methods of Calderón and Gustavsson-Peetre. Proc. Edinburgh Math. Soc. (2) 38(1995), No. 2, 261276.

3. T. Runst and W. Sickel, Sobolev spaces of fractional order, Nemytskij operators, and nonlinear partial differential equations. de Gruyter Series in Nonlinear Analysis and Applications, 3. Walter de Gruyter \& Co., Berlin, 1996.

4. K. TAIRA, Feller semigroups and degenerate elliptic operators. I, II. Conf. Semin. Mat. Univ. Bari Nos. 274, 275 (1999).

5. H. Triebel, Theory of function spaces. II. Monographs in Mathematics, 84. Birkhäuser Verlag, Basel, 1992.

6. W. P. Ziemer, Weakly differentiable functions. Sobolev spaces and functions of bounded variation. Graduate Texts in Mathematics, 120. Springer-Verlag, New York, 1989.

(Received 27.07.2004)

Authors' addresses:

T. Runst

Math. Institut, Friedrich-Schiller-University Jena

D-07740 Jena, Ernst-Abbe-Platz 1-4

Germany

E-mail: runst@minet.uni-jena.de

A. Youssfi

Laboratoire d'Analyse et de Mathématiques Appliquées

CNRS UMR 8050, Université Marne-La-Vallée

5 bd Descartes, Cité Descartes

Champs-sur-Marne, F-77454 Marne-La-Vallée Cedex 2

France

E-mail: youssfi@math.univ-mlv.fr 